\title{
COEFFICIENT INVERSE PROBLEM FOR THE DEGENERATE PARABOLIC EQUATION
}

\author{
NADIIA HUZYK
}

Abstract. The inverse problem for the degenerate parabolic equation is considered. The minor coefficient of the equation is a polynomial of the first power with respect to the space variable with unknown time-dependent coefficients. The conditions of local in time existence and global uniqueness of the classical solution to this problem are established. The case of weak power degeneration is investigated.

Mathematics subject classification (2020): 35R30, 35K65.

Keywords and phrases: Coefficient inverse problem, parabolic equation, weak power degeneration.

\section{REFERENCES}

[1] S. KABANIKHIN, Inverse and ill-posed problems: theory and applications, (2012), De Gruyter.

[2] M. Bertero, M. PiAnA, Inverse problems in biomedical imaging: Modeling and methods of solution, In book: Complex System in Biomedicine, (2006), 1-33.

[3] A. Lorenzi, E. PAPAROni, Direct and inverse problems in the theory of materials with memory, Rend. Sem. Univ. Padova. 87 (1992), 105-138.

[4] H. AzARI, C. Li, Y. NiE, S. Shang, Determination of an unknown coefficient in a parabolic inverse problem, Dynamics of Continuous, Discrete and Impulsive Systems. Series A: Math. Analysis. 11 (2004), 665-674.

[5] Taki-Eddine Oussaeif, Abdelfatah Bouziani, An inverse coefficient problem for parabolic equation under nonlocal boundary and integral overdetermination conditions, International Journal of Partial Differential Equation and Applications. 2, 3 (2014), 38-43.

[6] Mansur I. Ismailov, Bülent OǦUR, An inverse diffusion problem with nonlocal boundary conditions, Numerical Methods for Partial Differential Equations 32, 2 (2016), 564-590.

[7] J. R. Cannon, S. Peres-Esteva, Determination of the coefficient of $u_{x}$ in a linear parabolic equation, Inverse Problems 10, 3 (1993), 521-531.

[8] Yin Hong-Ming, Global solvability for some parabolic inverse problems, J. Math. Anal. and Appl. 162 (1991), 392-403.

[9] D. D. Trong, D. D. Ang, Coefficient identification for a parabolic equation, Inverse Problems $\mathbf{1 0}$, 3 (1994), 733-752.

[10] M. Dehghan, Parameter identification in a partial differential equation from overspecified data, Math. and Computer Modelling 41 (2005), 197-213.

[11] ELVIN I. AZIZBAYOV, The nonlocal inverse problem of the identification of the lowest coefficient and the right-hand side in a second-order parabolic equation with integral conditions, Bound Value Probl. 2019, 11 (2019), 1-19.

[12] M. S. Hussein, D. LESNIC, Identification of the Time-Dependent Conductivity of an Inhomogeneous Diffusive Material, Applied Mathematics and Computation 269 (2015), 35-58.

[13] M. I. Ismailov AND F. KANCA, The inverse problem of finding the time dependent diffusion coefficient of the heat equation from integral overdetermination data, Inverse Problems in Science and Engineering 20 (2012), 463-476.

[14] D. Lesnic, S. A. Yousefi And M. IVAnChov, Determination of a time-dependent diffusivity from nonlocal conditions, Journal of Applied Mathematics and Computing 41 (2013), 301-320. 
[15] M. S. Hussein, D. LeSnic, V. KAmynin And A. Kostin, Direct and inverse source problems for degenerate parabolic equations, Journal of Inverse and Ill-posed Problems 28, 3 (2020), 425-448.

[16] G. Di Blasio, A. Lorenzi, An Identification Problem in Age-Dependent Population Diffusion, Numerical Functional Analysis and Optimization 34, 1 (2013), 36-73.

[17] B. Kaltenbacher, M. Klibanov, An Inverse Problem for a Nonlinear Parabolic Equation with Applications in Population Dynamics and Magnetics, SIAM J. Math. Anal. 39, 6 (2008), 1863-1889.

[18] N. SALDINA, An inverse problem for a generally degenerate heat equation, J. of Lviv Polytechnic National University. Physical and Math. Sciences 566 (2006), 59-67.

[19] M. IVANChOV AND N. SALDINA, An inverse problem for strongly degenerate heat equation, J. Inv. Ill-Posed Problems. 14, 5 (2006), 465-480.

[20] N. HRYNTSIV, An inverse problem for a strongly power degenerate parabolic equation in a free boundary domain, Visnyk Lviv. Univ. Ser. Mech.-Math. 64 (2007), 84-97, (in Ukrainian).

[21] N. HRYNTSIV AND M. IVANCHOV, Inverse problem for the strongly degenerate heat equation in a domain with free boundary, Ukrain. Math. J. 61, 1 (2009), 30-49.

[22] N. HRYNTSIV, The inverse problem with free boundary for a weakly degenerate parabolic equation, J. of Math. Sciences 183, 6 (2012), 779-795.

[23] M. IVAnchov, A. Lorenzi AND N. Saldina, Solving a scalar degenerate multidimensional identification problem in a Banach space, J. Inv. Ill-Posed Problems 16 (2008), 1-19.

[24] N. HUZYK, Inverse problem of determining the coefficients in a degenerate parabolic equation, Electron. J. Diff. Eq. 2014, 172 (2014), 1-11.

[25] N. HUZYK, Determination of the Lower Coefficient in a Parabolic Equation with Strong Power Degeneration, Ukr. Math. J. 68, 7 (2016), 1049-1061.

[26] P. Cannarsa, J. Tort And M. Yamamoto, Determination of source terms in a degenerate parabolic equation, Inverse Problems 26, 10 (2010), 105003.

[27] LI RANRAN AND LI ZHIYUAN, Identifying unknown source in degenerate parabolic equation from final observation, Inverse Problems in Science and Engineering, (2020), doi:10.1080/17415977.2020.1817005.

[28] M. IVANCHOV, Inverse problems for equations of parabolic type, (2003), Lviv: VNTL Publishers.

[29] O. A. Ladyzhenskaya, V. A. Solonnikov, and N. N. Uraltseva, Linear and Quasilinear Equations of Parabolic Type, (1968), American Mathematical Society, Providence, Rhode Island, USA. 